\title{
Wireless non-invasive continuous respiratory monitoring with FMCW radar: a clinical validation study
}

\author{
K. van Loon ${ }^{1}$ M. J. M. Breteler ${ }^{1}$ L. van Wolfwinkel ${ }^{1} \cdot$ A. T. Rheineck Leyssius ${ }^{2}$. \\ S. Kossen ${ }^{3}$ C. J. Kalkman ${ }^{1}$ B. van Zaane ${ }^{1}$ L. M. Peelen ${ }^{1,4}$
}

Received: 1 May 2015/ Accepted: 16 September 2015/Published online: 30 September 2015

(c) The Author(s) 2015. This article is published with open access at Springerlink.com

\begin{abstract}
Altered respiratory rate is one of the first symptoms of medical conditions that require timely intervention, e.g., sepsis or opioid-induced respiratory depression. To facilitate continuous respiratory rate monitoring on general hospital wards a contactless, noninvasive, prototype monitor was developed using frequency modulated continuous wave radar. We aimed to study whether radar can reliably measure respiratory rate in postoperative patients. In a diagnostic cross-sectional study patients were monitored with the radar and the reference monitor (pneumotachograph during mechanical ventilation and capnography during spontaneous breathing). Eight patients were included; yielding $796 \mathrm{~min}$ of observation time during mechanical ventilation and
\end{abstract}

A brief summary of this work was presented at the Dutch anesthesiology research meeting ("NVA Wetenschapsdag") 19/9/14 in Zeist, the Netherlands. The abstract was also presented at the ASA congress $14 / 10 / 14$ in New Orleans, USA.

Electronic supplementary material The online version of this article (doi:10.1007/s10877-015-9777-5) contains supplementary material, which is available to authorized users.

K. van Loon

K.vanLoon-3@umcutrecht.nl

1 Department of Anesthesiology, University Medical Center Utrecht, Mailstop Q 04.2.313, P.O. Box 85500, 3508 GA Utrecht, The Netherlands

2 Department of Intensive Care, ZGT Hospital, Almelo, The Netherlands

3 Radar Technology at TNO, The Hague, The Netherlands

4 Julius Center for Health Sciences and Primary Care, University Medical Center Utrecht, Utrecht, The Netherlands
521 min during spontaneous breathing. After elimination of movement artifacts the bias and $95 \%$ limits of agreement for mechanical ventilation and spontaneous breathing were $-0.12(-1.76$ to 1.51$)$ and $-0.59(-5.82$ to 4.63 ) breaths per minute respectively. The radar was able to accurately measure respiratory rate in mechanically ventilated patients, but the accuracy decreased during spontaneous breathing.

Keywords Radar $\cdot$ Respiratory rate $\cdot$ Monitoring · Remote

\section{Introduction}

Several observational studies support the idea that in-hospital deaths can be prevented by early recognition of abnormal vital signs as a marker of physiological decline [1-3]. However, timely recognition of abnormal vital signs on general hospital wards remains a major challenge as nurses have only limited time to observe their patients and perform systematic evaluations. Changes in respiratory rate (RR) are an important indicator of physiological decline and were found to be present as early as 6-24 $\mathrm{h}$ prior to adverse events such as cardiac arrest [2-6]. Also, altered $\mathrm{RR}$ is one of the first symptoms of numerous diseases that could benefit from timely intervention, hereby preventing further deterioration. Examples are increased RR in pneumonia, sepsis and cardiogenic shock; and decreased RR with opioid overdose. Nevertheless, clinicians still underestimate the importance of a change in RR and it is probably the least observed and recorded vital sign on general hospital wards [7]. Currently, the usefulness of RR is limited due to its large inter-observer variability, as RR is counted by observing chest wall movements [8, 9]. More 
importantly, observers (i.e. nurses) can only provide intermittent assessments, as RR is typically estimated only two or three times a day. This leaves a patient's respiration unmonitored for long periods of time (typically $8 \mathrm{~h}$ or more) and any physiological decline that occurs between these RR observations can easily go unnoticed.

Recently, the Opal radar system was developed to detect vital signs (RR and heart rate) in humans (TNO laboratories, The Hague, Netherlands). The radar uses frequency modulated continuous wave (FMCW) radar to obtain a periodic signal by frequency modulating a continuous signal that is mixed with an echo. The radar is therefore able to obtain information on movement in multiple directions (e.g. the expansion of the chest during breathing), based on the principle of integrating a transmitted and received signal [10]. The radar system was previously found to be capable of detecting breathing patterns noninvasively in human volunteers [10]. However, rigorous scientific evaluation has to be conducted before introducing a new technical device into clinical practice. The primary aim of this study was therefore to determine whether this prototype radar system is able to reliably measure RR in patients during mechanical ventilation and spontaneous breathing.

\section{Materials and methods}

\subsection{Study population}

We aimed to study the FMCW radar in postoperative surgical patients during mechanical ventilation (MV), where RRs and tidal volumes are exactly known, and during spontaneous breathing. We included adult patients at the University Medical Center Utrecht, the Netherlands, who underwent elective robotic assisted laparoscopic prostatectomy or hysterectomy and received post-operative ventilation on the postoperative anesthesia care unit (PACU) (as per clinical protocol). Patients with an implantable cardiac device, or on renal replacement therapy, or who refused participation or who were unable to give informed consent, were excluded. The institutional review board (IRB) of the University Medical Center Utrecht reviewed the study protocol and found that it was not subject to the Dutch act on "medical research involving human subjects". Therefore, the IRB waived the need for informed consent, but we decided to request written informed consent from all participants in order to promote transparent information provision to patients. The study was conducted in accordance with the moral, ethical, and scientific principles governing clinical research as set out in the Declaration of Helsinki [11] and good clinical practice.

\subsection{Study design and measurements}

This study was a diagnostic cross-sectional observational study in which patients were monitored after surgery during recovery in the PACU to conform with current guidelines for postoperative care. The non-invasive FMCW radar signals (with the device mounted to the ceiling above the patient, see Fig. 1) were recorded simultaneously with those from the reference standard. During MV the reference standard was the pneumotachograph from the ventilator (Raphael, Hamilton Medical AG 2005, Bonaduz, Switzerland). The pneumotachograph has an accuracy of $\pm 1 \mathrm{breath} / \mathrm{min}$. During spontaneous breathing the reference standard was a capnograph (Compact S5, Datex Ohmeda, Inc., Helsinki, Finland) with end-tidal carbon dioxide (etCO2) sampling lines (Smart CapnoLine Plus, Oridion Medical 1987 Ltd, Jerusalem, Israel). The capnograph has an accuracy of \pm 1 breath/min in the range 4-20 breaths/ min. In addition, patients were observed during study measurements by one of the authors $(\mathrm{KvL})$, who recorded time-stamped patient movements and staff activity around the bed. Nursing and medical staff was blinded to the study measurements with the FMCW radar.

\subsection{FMCW radar principle}

The FMCW radar system is a small box sized 7.5 by $10 \mathrm{~cm}$ mounted to the ceiling above the bed. This prototype radar operates on a frequency of $9-10 \mathrm{GHz}$ with an effective isotopically radiated power of $14 \mathrm{~mW}$, which is well within restrictions for human exposure to electromagnetic fields [12]. The emitted electromagnetic radio waves (frequency of $9.5 \mathrm{GHz}$ ) propagate to the patient, are reflected, and arrive back at the radar with a time difference $(\Delta t)$. This causes a frequency difference between emitted and received signal due to the fact that the radar changes the frequency of the radio waves over time, consistent with a sawtooth pattern in Fig. 1. This frequency shift $(\Delta f)$ over time enables us to measure the time difference between emitted and received signal $(\Delta t)$, and obtain the distance $(R)$ between the radar and the patient. The relation between distance and time difference is given in the formula in Fig. 1. Patient breathing (or moving) changes the amount of reflected energy to the radar (resulting in amplitude changes in the radar output signal) and the distance to the patient (resulting in phase changes in the radar output signal). Additional open source technical information about FMCW radar is available online [13] and in Supplement 1.

\subsection{Signal analysis}

The raw data recorded by the FMCW radar system consisted of numerical indicators for the amplitude and phase 
Fig. 1 The principle of frequency modulated continuous wave (FMCW) radar. The frequency shift $(\Delta f)$ of the emitted radar waves over time enables us to determine the distance to the patient. Patient breathing (or movement) changes the amplitude (energy) of the reflected signal (the red line) and the distance $(R)$

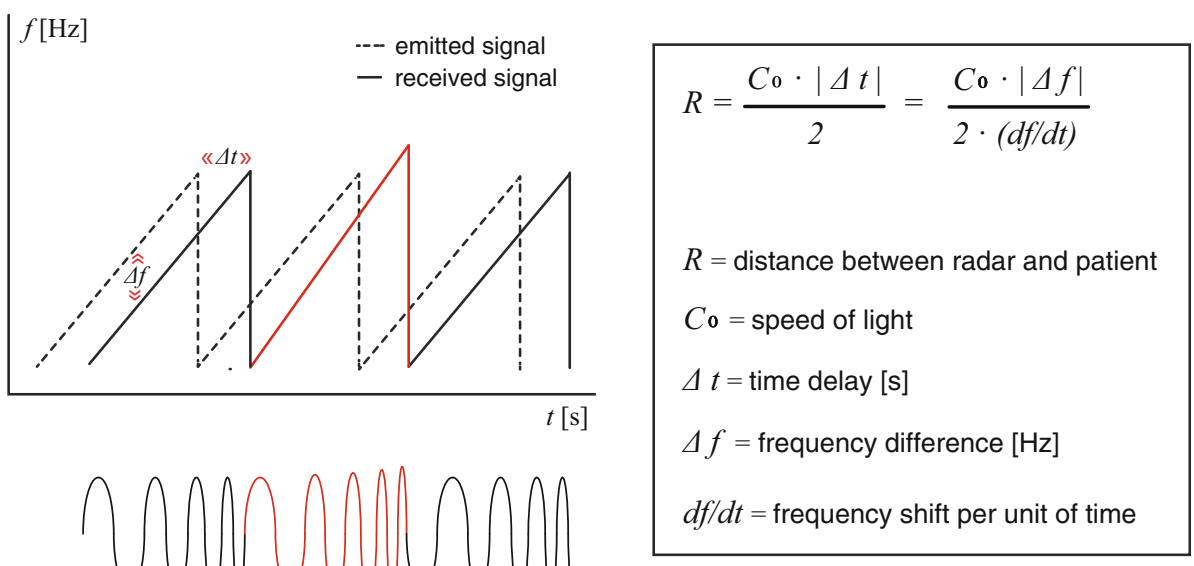

from a certain spatial range $R$. The area under the radar was divided into horizontal planes (range bins) of $21 \mathrm{~cm}$ height. Patient movement (e.g. chest wall movement for respiration) typically extended over 2 or 3 range bins. By selecting the range bin with the highest power in the frequency band up to $40 \mathrm{~Hz}$, artifacts were largely neglected and the optimal range bin was selected. As the patient could change position several times, the 'optimal' range bin was selected every minute. The amplitude and phase of the echoed radar waves contained information about movement of the patient inside the selected range bin. In this study we only used the amplitude information.

RRs were determined with Fast Fourier Transform (FFT) [14]. As we aimed to conduct our analysis both on raw data (complete dataset) and after removal of voluntary movement artifacts (artifact reduced dataset), a dedicated algorithm was written for artifact detection. We first visually identified differences between consecutive points during rest and during other activities, using the observations on patient and staff movement. Subsequently, a fixed threshold (electric potential of $0.005 \mathrm{~V}$ ) was applied for differences between consecutive amplitude points. The number of artifacts was defined as the number of points that exceeded this threshold. During MV FFT was applied on each 60-s time sample, as the reference standard was also retrieved from a 60-s time sample, to obtain the corresponding $\mathrm{RR}$ by taking the maximum peak in the frequency window. During spontaneous breathing FFT was applied over a $30 \mathrm{~s}$ time interval, as it was expected that during spontaneous breathing the number of artifacts would increase due to activities of nursing staff or obvious patient movement. Out of two retrieved RRs, the one with the lowest number of artifacts was used for further analysis. In case both RRs had a similar amount of artifacts, the RR used in further analysis was calculated as the average of these RRs. For the reference standards we did not develop a specific algorithm for artifact detection, but used the RRs as provided by the pneumotachograph and the capnograph respectively.

\subsection{Outcomes}

The primary outcome was the RR. As bradypnoea is a serious life threatening condition with a narrow diagnostic window, we considered a RR with limits of agreement (LoA) of -2 to 2 breaths per minute acceptable. The secondary outcome was accuracy in detecting abnormal respiration ( $\mathrm{RR}<12$ or $>20$ breaths per minute) according to the early warning score definitions [15].

\section{Statistics}

The paired time series of RR measurements (one data point every minute) derived from FMCW radar and the reference standard were compared using the Bland and Altman method for repeated measurements [16]. In this method the variance for differences between the average difference across patients is corrected for the number of measurements per patient. We determined the bias (mean difference) and $95 \%$ LoA's $( \pm 1.96 * \mathrm{SD})$ for the complete and reduced (after removal of artifacts) dataset. For this type of studies no formal rules for sample size calculations are available. We considered 250 measurement pairs for respiratory rate to be sufficient for adequate estimation of the repeated measures Bland-Altman bounds. Abnormal breathing rates were defined as a respiratory rate below 12 breaths and above 20 breaths per minute. Sensitivity, specificity, positive predictive value, and negative predictive value for abnormal breathing were calculated. Corresponding confidence intervals were produced with the Wilson Score method without continuity correction. 
Additionally, a Clarke error grid analysis (EGA) was performed to quantify clinical accuracy and the consequences for clinical decision making [17]. The analyses were conducted using MatLab (The MathWorks ${ }^{\circledR}$, Inc) and the public domain statistical software ' $R$ ' version 2.15.3 (URL: http://www.R-project.org).

\section{Results}

From May 2011 to August 2011, 796 min were recorded during MV and 521 min during spontaneous breathing in eight patients who gave informed consent for study participation. For MV, 796 complete RR measurement pairs were available, and 351 (67\%) for spontaneous breathing. Missing data of spontaneous breathing measurements consisted of 169 min with missing capnography measurements and one missing FMCW radar measurement. The number of observations on each patient ranged from 17 to 170 during MV and 23-70 during spontaneous breathing. Characteristics for the individual patients are shown in Table 1 . None of the patients had pulmonary comorbidities or abnormalities of the chest wall. All patients received analgesia with acetaminophen and intravenous morphine for postoperative pain during their stay on the PACU. During spontaneous breathing patients received supplemental oxygen (1-3 L/min). In $98.3 \%$ of all $\mathrm{SpO} 2$ readings the $\mathrm{SpO} 2$ was $\geq 95 \%$, and none of the patients had $\mathrm{SpO} 2$ readings below $90 \%$.

\subsection{Respiratory rate during mechanical ventilation}

Bias and $95 \%$ LoA from comparisons between the radar and the pneumotachograph are corrected for the number of observations on each patient and shown in Table 2. After artifact removal during MV, 441 min could be analyzed. The bias (RR radar minus RR pneumotachograph) was -0.12 breaths per minute with a $95 \%$ LoA of -1.75 to 1.51 breaths per minute. Bland and Altman plots for the complete and artifact reduced datasets are shown in respectively Fig. 2a and b.

\subsection{Respiratory rate during spontaneous breathing}

During spontaneous breathing, after artifact removal, the RR measured by the radar was on average -0.59 breaths per minute with a $95 \%$ LoA of -5.82 to 4.63 breaths per minute (Table 2). Bland and Altman plots for the complete and reduced dataset displayed in Fig. 3 indicate that the difference between FMCW radar and capnography depends on the average breathing rate. For respiratory rates below 10 and above 15 breaths per minute differences between FMCW radar and capnography are within the $95 \%$ LoA, whereas between 10 and 15 breaths per minute the monitors differ considerably

\subsection{Diagnostic accuracy for abnormal breathing}

Tachypnoea was defined as a RR above 20 breaths per minute. During spontaneous breathing only $2 \mathrm{~min}$ with tachypnoea were recorded by the capnograph. Therefore we were unable to calculate diagnostic accuracy for tachypnoea. Bradypnoea occurred frequently during spontaneous breathing, a RR below 12 breaths per minute was present in $64 \%$ of all RR minutes in the complete dataset analysis. The sensitivity, specificity, PPV and NPV are shown in Table 3. After removal of artifacts, the FMCW radar had a positive predictive value (PPV) of $86 \%$ (79-92\%), and a negative predictive value (NPV) of $75 \%$ (64-85\%) for detection of bradypnoea. Ninety-one percent

Table 1 Characteristics of individual patients and measurements during monitoring with frequency modulated continuous wave radar in postoperative patients

\begin{tabular}{|c|c|c|c|c|c|c|c|c|c|}
\hline \multirow[b]{2}{*}{ Patient } & \multirow[b]{2}{*}{$\begin{array}{l}\text { Gender } \\
(\mathrm{m} / \mathrm{f})\end{array}$} & \multirow[b]{2}{*}{$\begin{array}{l}\text { Age } \\
\text { (years) }\end{array}$} & \multirow[b]{2}{*}{$\begin{array}{l}\text { BMI } \\
\left(\mathrm{kg} \mathrm{m}^{2}\right)\end{array}$} & \multirow[b]{2}{*}{$\begin{array}{l}\text { Surgical } \\
\text { procedure }\end{array}$} & \multicolumn{2}{|c|}{ Mechanical ventilation } & \multicolumn{3}{|c|}{ Spontaneous breathing } \\
\hline & & & & & $\begin{array}{l}\text { Tidal volumes } \\
\text { during MV } \\
\text { mean }(\mathrm{SD})^{\mathrm{a}}\end{array}$ & $\begin{array}{l}\text { Radar } \\
\text { measures } \\
(\min )\end{array}$ & $\begin{array}{l}\text { Reference } \\
\text { measures } \\
(\min )\end{array}$ & $\begin{array}{l}\text { Radar } \\
\text { measures } \\
(\min )\end{array}$ & $\begin{array}{l}\text { Reference } \\
\text { measures } \\
(\mathrm{min})\end{array}$ \\
\hline 1 & M & 53 & 26.0 & Prostatectomy & $768(48)$ & 112 & 112 & 31 & 30 \\
\hline 2 & M & 54 & 31.2 & Prostatectomy & 903 (199) & 141 & 141 & 26 & 23 \\
\hline 3 & M & 62 & 24.5 & Prostatectomy & $697(20)$ & 58 & 58 & 60 & 56 \\
\hline 4 & M & 58 & 24.5 & Prostatectomy & $695(67)$ & 170 & 170 & 42 & 37 \\
\hline 5 & $\mathrm{~F}$ & 34 & 31.7 & Hysterectomy & $586(86)$ & 156 & 156 & 77 & 67 \\
\hline 6 & $\mathrm{~F}$ & 36 & 19.4 & Hysterectomy & $651(82)$ & 72 & 72 & 102 & 34 \\
\hline 7 & M & 63 & 25.3 & Prostatectomy & $678(110)$ & 17 & 17 & 143 & 70 \\
\hline 8 & $\mathrm{~F}$ & 58 & 22.7 & Hysterectomy & 413 (49) & 70 & 70 & 39 & 34 \\
\hline
\end{tabular}

$M$ male, $F$ female, Surgical procedures were robot assisted laparoscopic procedures

${ }^{a} M V$ mechanical ventilation 
Table 2 Primary outcome: respiratory rates during frequency modulated continuous wave radar monitoring in postoperative patients

\begin{tabular}{llccc}
\hline Study phase and analysis & $\begin{array}{l}\text { Number of } \\
\text { measurement pairs }\end{array}$ & Bias & SD & $\begin{array}{l}\text { Lower } \\
95 \% \text { LoA }^{\mathrm{b}}\end{array}$ \\
\hline $\begin{array}{l}\text { Mechanical ventilation } \\
\text { Complete dataset }\end{array}$ & 796 & -0.37 & 1.64 & $\begin{array}{l}\text { Upper } \\
95 \% \text { LoA }^{\mathrm{b}}\end{array}$ \\
Reduced dataset $^{\mathrm{a}}$ & 441 & -0.12 & 0.83 & -3.58 \\
Spontaneous breathing & & & & -1.76 \\
Complete dataset $^{\text {Reduced dataset }}$ & 351 & -1.21 & 3.57 & -8.20 \\
\hline
\end{tabular}

${ }^{\text {a }}$ Dataset after elimination of movement artifacts

b $L o A$ limits of agreement
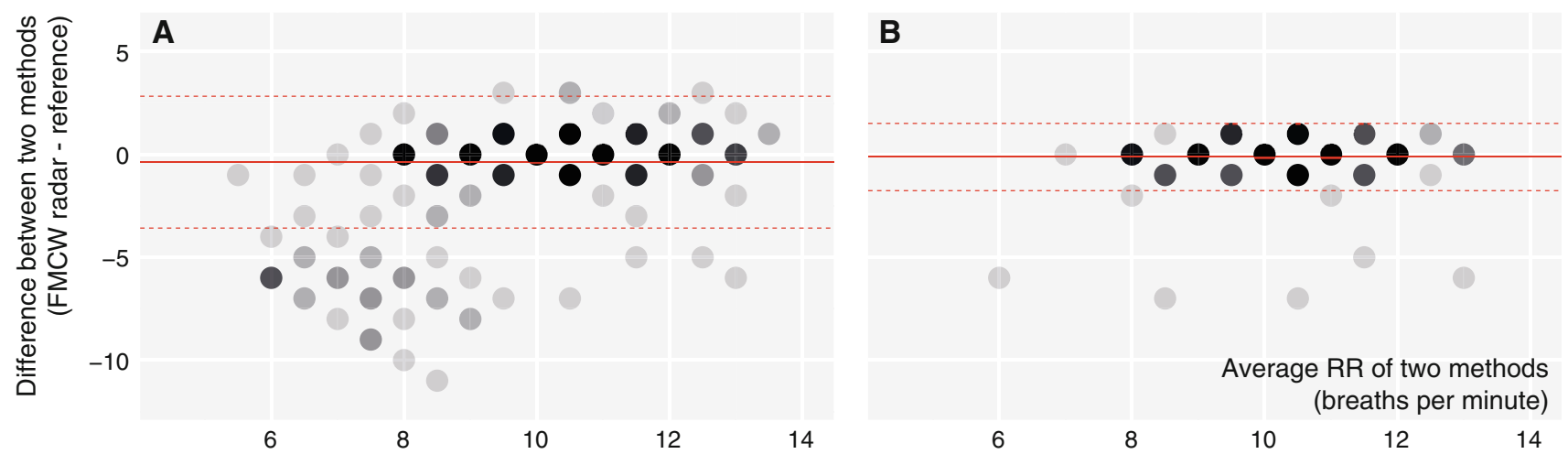

Fig. 2 Bland and Altman plots for complete (a) and movement artifact reduced (b) datasets for respiratory rate during mechanical ventilation with 1 (filled circle) to 10 (filled circle) measurement pairs
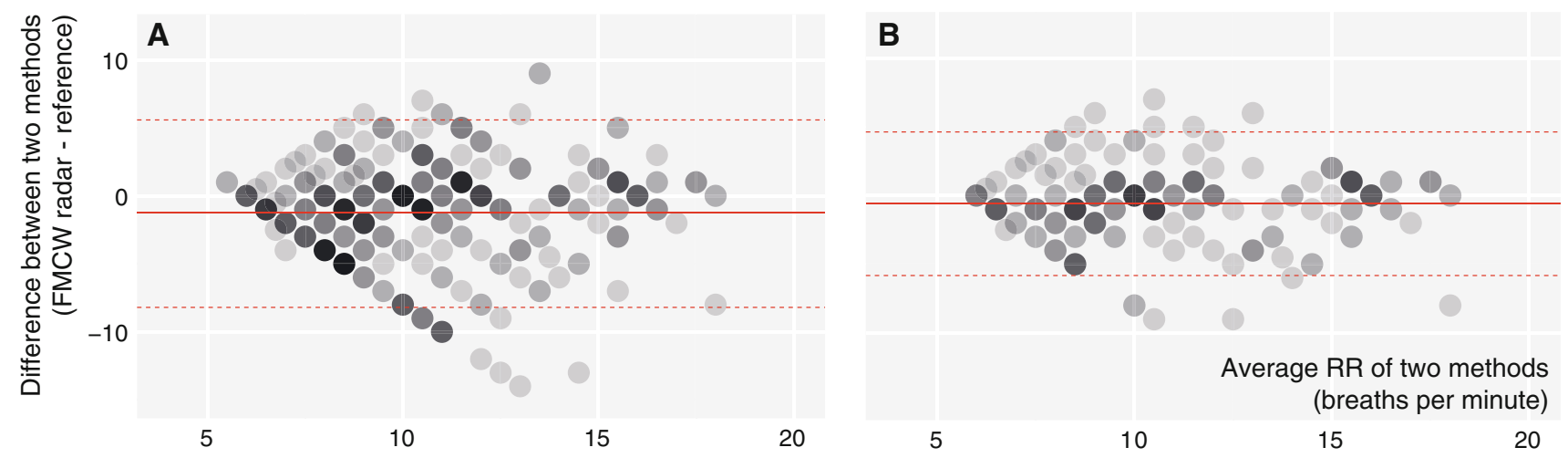

Fig. 3 Bland and Altman plots for complete (a) and movement artifact reduced (b) datasets with respiratory rate during spontaneous breathing with 1 (filled circle) to 10 (filled circle) measurement pairs

of respiratory rate measurements in the error grid analysis are within region $\mathrm{A}$ and $\mathrm{B}$ (Fig. 4), respectively within $20 \%$ of the reference measurement, or outside $20 \%$ of the reference but not leading to unnecessary treatment. Region $\mathrm{D}$, indicating a potentially dangerous failure to detect bradypnoea contains nine percent of the measurements. None of the measurements were in region $\mathrm{C}$ or $\mathrm{E}$, which means that none of the measurements would lead to unnecessary treatment or confusion between tachypnoea and bradypnoea.

\section{Discussion}

We studied the ability of a prototype FMCW radar system to measure RR in patients during MV and during spontaneous breathing. After elimination of obvious movement 
Table 3 Secondary outcome: diagnostic accuracy for bradypnoea (respiratory rate $<12$ breaths per minute) during spontaneous breathing

\begin{tabular}{|c|c|c|c|c|c|c|c|c|}
\hline Study analysis & $\begin{array}{l}\text { True } \\
\text { positives }\end{array}$ & $\begin{array}{l}\text { False } \\
\text { positives }\end{array}$ & $\begin{array}{l}\text { True } \\
\text { negatives }\end{array}$ & $\begin{array}{l}\text { False } \\
\text { negatives }\end{array}$ & $\begin{array}{l}\text { Sensitivity } \\
(95 \% \mathrm{CI})\end{array}$ & $\begin{array}{l}\text { Specificity } \\
(95 \% \text { CI })\end{array}$ & $\begin{array}{l}\mathrm{PPV}^{\mathrm{c}} \\
(95 \% \mathrm{CI})\end{array}$ & $\begin{array}{l}\mathrm{NPV}^{\mathrm{d}} \\
(95 \% \mathrm{CI})\end{array}$ \\
\hline $\mathrm{SB}^{\mathrm{a}}$ (complete dataset) & 180 & 56 & 69 & 45 & $80(74-85)$ & $55(46-64)$ & $76(71-81)$ & $61(51-69)$ \\
\hline $\mathrm{SB}^{\mathrm{a}}\left(\right.$ reduced dataset $\left.{ }^{\mathrm{b}}\right)$ & 104 & 17 & 48 & 16 & $87(80-92)$ & $74(63-84)$ & $86(79-92)$ & $75(64-85)$ \\
\hline
\end{tabular}

Values are number and proportion

${ }^{\text {a }} S B$ spontaneous breathing

${ }^{\mathrm{b}}$ Dataset after elimination of movement with artifacts due to obvious movement

c $P P V$ positive predictive value

d $N P V$ negative predictive value

Fig. 4 Clarke error grid to quantify clinical accuracy of the respiratory rate measurements by radar. Region $(E)$ represent those points where tachypnoea and bradypnoea are confused, region $(D)$ indicates a potentially dangerous failure to detect bradypnoea or tachypnoea, in region $(C)$ are points leading to unnecessary treatment, region $(B)$ contains points outside $20 \%$ of the reference but not leading to unnecessary treatment, region (A) are points within $20 \%$ of the reference measurement

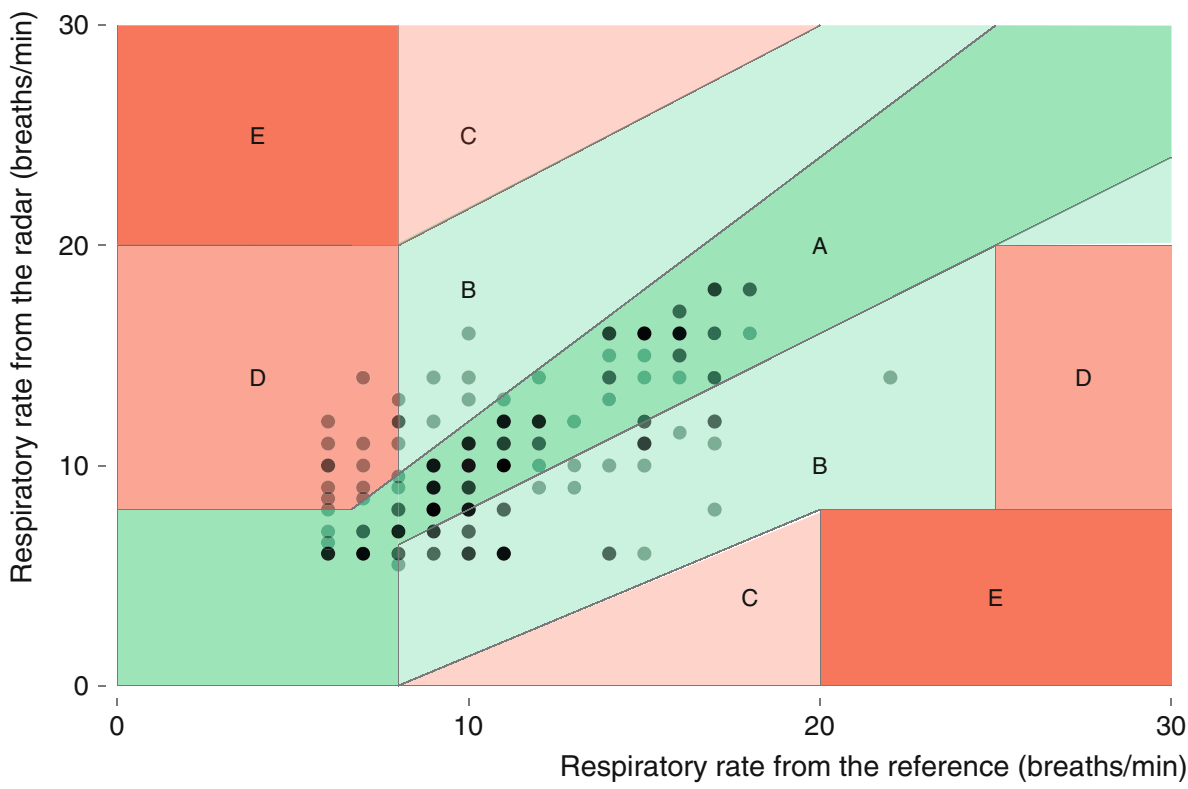

artifacts, the FMCW radar accurately measured RR in patients during MV with a deviation within two breaths of the reference standard. During spontaneous breathing, while the patient is awake and physically active, the accuracy of the FMCW radar to detect RR every minute was outside our acceptable limits. Presumably, the clinical study setting, the pharmacological (e.g. opioid administration) and the surgical treatment, all contributed to the differences between the FMCW radar- and capnography RR measurements during the spontaneous breathing phase. Interestingly, the deviation of the FMCW radar RR measurements strongly depended on the average RR. The radar appeared to be more accurate during episodes with low RR. One possible explanation is that patients in deep sleep, shortly after anesthesia and after opioid administration, lie still and breathe slowly. In general, patients with an opioid breathing pattern show bradypnoea with large tidal volumes and larger chest wall movement [18]. This probably explains the high accuracy of FMCW radar during episodes with low breathing rates, with a sensitivity of $87 \%$ as well as a positive predictive value of $86 \%$ for detection of bradypnoea. This finding is very important, since during bradypnoea even small deviations in RR measurements are clinically relevant.

The accuracy of the FMCW radar improved when we excluded 1-min epochs with obvious movement artifacts. These artifacts were characterized by a large fluctuation of the reflected energy (amplitude) within a least five range bins (Supplement 2). Thus, the FMCW radar is not able to detect RR accurately when there is obvious patient (or provider) movement in the same range bin as the chest wall movements. When considering how these artifacts may influence the feasibility of FMCW radar to monitor RR in patients on a general hospital ward, there are several 
scenarios to consider. When a caregiver or family member in close vicinity of the patient is responsible for the movement artifacts, this person will probably notice apnea/ unresponsiveness, if present. Also, when radar artifacts are caused by active patient movements, these artifacts imply that the patient is alive and breathing. It should be noted however that there are, two possibly life-threatening exceptions to this latter situation: extreme agitation due to hypoxemia and grand mal seizure activity. Furthermore, with any technique that relies on measuring chest wall movements, detecting such movement does not guarantee displacement of air. Therefore the FMCW radar might have difficulty recognizing progressive upper airway obstruction. In this specific situation chest wall movements will be intact without air movement. This problem can potentially be solved if future radar algorithms are optimized to detect paradoxical movement of chest wall and abdomen, and other opioid- induced changes of the breathing pattern (e.g. increased intercostal contribution, increased tidal volume, and increased respiratory variability) $[18,19]$ by pattern recognition techniques [20].

As far as we know, this is the first study with FMCW radar to measure RR in postoperative surgical patients. A variety of medical radars to measure $R R$ and heart rate have been described. Most of these studies used continuous wave (CW) Doppler radar or impulse ultra wide band (UWB) radars. See for a detailed overview the FFI report Medical Radar literature overview by Aardal [21]. Although CW radars are sensitive in detecting time varying physiological phenomena, they do not provide range information which FMCW and UWB radars do provide. While some of these radars are studied in hospital environments, most experiments were conducted under controlled laboratory conditions. Vasu [22] studied RR overnight with a non-contact Doppler radar in ten patients without sleep disorders and compared these measurements to expert annotations. Other (UWB) radars that are being tested in hospital environments showed correct heart rate and RR measurements [23]. In our study only eight patients were included, but we provide a large number of data points during both MV (796 data points) and spontaneous breathing (351 data points) with both the FMCW radar and the reference device.

\section{Limitations}

When interpreting the findings of this study some limitations should be taken into account. First, since a predefined algorithm for analyzing FMCW radar data was not available, we may have selected a suboptimal signal-processing algorithm. In this study we used FFT to translate the amplitude information of the radar waves into RRs. FFT uses the symmetry and periodicity of the signal to create a frequency window. Unlike during MV, the breathing pattern during spontaneous breathing in awakening patients is variable, even more evident than in awake patients, with alternately deep and shallow breaths, and fast and slow breathing (dependent on alertness, speech and emotional state). Probably, this is also the case for the observed RR differences in the range 10-15 breaths per minute. In that situation, the peak energy in the frequency domain will disperse into a wider frequency band, making 'exact' RR estimation difficult and potentially unreliable. Alternative signal processing techniques as wavelet transform (WT) could be used in future evaluations [24]. We cannot exclude that this may improve the performance of FMCW radar. Another possible limitation of the current algorithm is the selection of the 'optimal' range bin depending on the highest power per range bin. Range bins with high frequencies (i.e. due to artifacts) were excluded before selection of the optimal range bin. It is conceivable that this selection order may have induced suboptimal range bin selection in some situations. Furthermore, for certain range bins the amplitude of the breathing signal shows two maxima per time period, that could indicate that different body parts move inside the same range bin, or that the patient oscillates through the center of the range bin, impeding the extraction of the correct peak in the frequency window. Another restriction of the signal analysis is that the literature is silent on thresholds for the detection of artifacts. We chose a fixed artifact threshold at $0.005 \mathrm{~V}$ based on the amplitude waveforms during observed movements. A lower threshold $(<0.005 \mathrm{~V})$ could eliminate more artifacts at the expense of limiting the number of available valid RR measurements. On the other hand, improved artifact elimination analysis could result in a higher proportion of epochs with reliable RR estimations and lower rate of false positive alarms. This is especially important in low care settings such as general hospital wards with lower nurse-to-patient ratio than recovery rooms or intensive care units.

A second limitation of our study was that our approach of rejecting 1-min epochs with excessive artifacts decreased the availability of continuous RR monitoring (Supplement 3). This procedure also made the relation between consecutive RR measurements more variable, which potentially hinders interpretation of the results from the Bland and Altman plots. For respiratory monitoring in clinical practice, slightly variable RR measurement updates are probably not detrimental, because the goal for continuous monitoring is to recognize physiological decline, which typically develops in the hours preceding a serious adverse event.

Third, it is important to consider that FMCW radar information consists of a complex time signal with two 


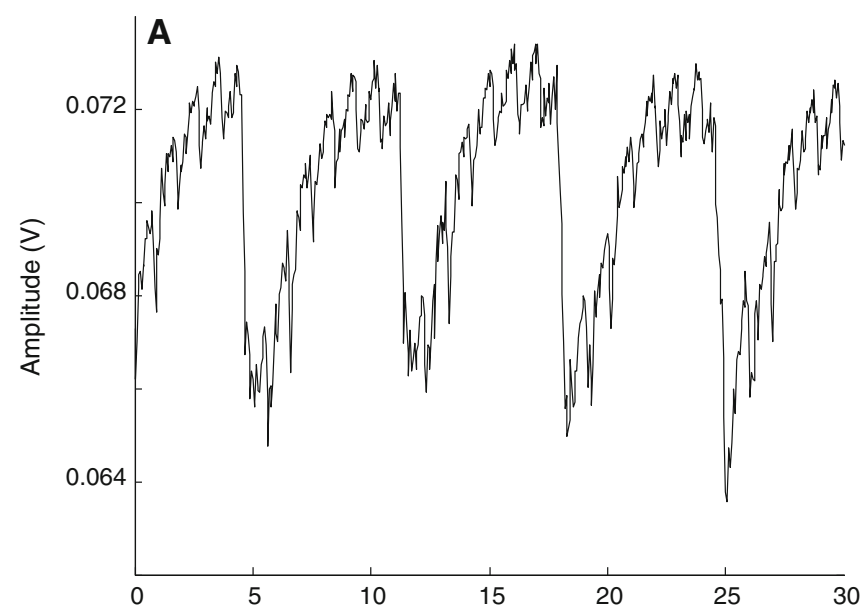

Fig. 5 Potential features of the frequency modulated continuous wave radar. a Example (patient number 8) of five breaths with 31 superimposed heartbeats on the $30 \mathrm{~s}$ radar signal during mechanical ventilation, that correspond to the heart rate measured by pulse oximetry. b Example (patient number 1) of a radar trace during $50 \mathrm{~s}$ of spontaneous breathing with different breathing patterns potentially

dimensions describing the echoed radar waves, namely the amplitude and phase. In this study we used only the amplitude information to estimate RRs. Incorporating the phase information as a second dimension could give additional information and can potentially increase the accuracy of the RR estimation in future studies.

\subsection{Other potential applications}

Although our study focused on detection of RR, we noticed that the heart rate was present in some parts of the amplitude information. Figure 5a shows an example of five breaths with 31 superimposed beats on the $30 \mathrm{~s}$ radar signal. This corresponds with the patients' actual heart rate of 63 beats per minute during this specific period. This is in line with findings of Aardal [25] who demonstrated the ability of FMCW radar to detect heart rate non-invasively. Future studies should determine if heart rate can be reliably determined from the raw radar data using optimized signal processing algorithms.

Another interesting issue is whether the raw amplitude radar data can also be used to estimate respiratory depth. Being able to distinguish between progression to rapid shallow breathing versus rapid deep breathing would be clinically important to assess a patient's reserve capacity. Some patients had radar amplitude patterns that suggested this might be the case (Fig. 5b). Estimating actual tidal volumes is likely to be much more difficult, since a patient in the supine position will produce different radar amplitudes compared to the lateral position. This problem may be solved by using two different radars placed on the

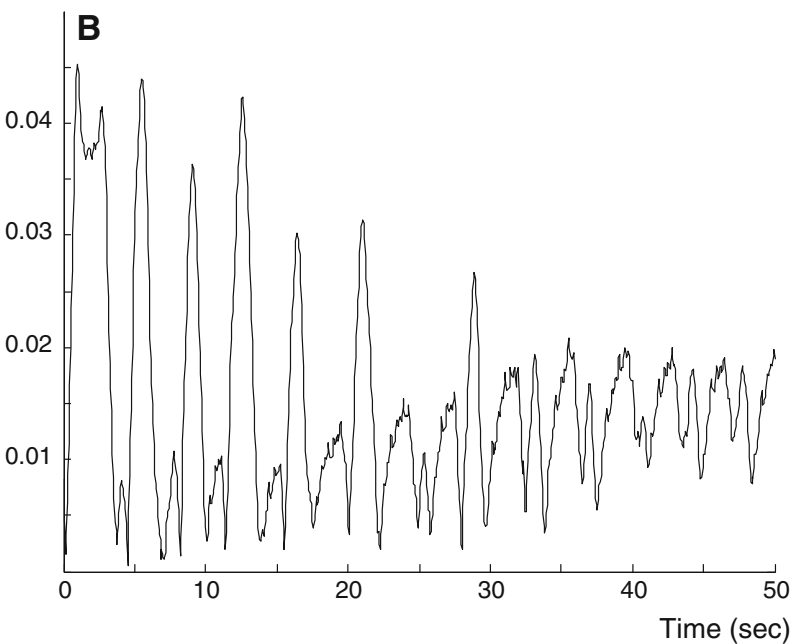

corresponding to the tidal volumes. Every inspiratory cycle is characterized by a first peak (chest wall expansion during inhalation), followed by a more smoothed peak (during exhalation). We presume that larger tidal volumes, thus increased chest wall expansion result in higher amplitudes of the echoed radar waves

ceiling above the patient instead of just one. Both phenomena are subject to future study.

\section{Conclusion}

Considering the ability of the FMCW radar system to accurately track RR during MV accurately and the possibilities for further optimizing of the signal processing algorithms, we suggest that future iterations of this wireless technique have the potential to support early recognition of physiological decline in spontaneously breathing patients on general hospital wards. However, the accuracy of RR determination during spontaneous breathing needs further refinement. Nonetheless, even with the current relatively crude movement artifact removal algorithm, enough epochs were left to track the RR trend of spontaneously breathing postoperative patients recovering from general anesthesia on a minute to minute basis. To confirm the feasibility of this radar-based technique for continuous and wireless respiratory monitoring in patients on a general hospital ward, a larger prospective cohort study is needed.

Acknowledgments Fokko P. Wieringa, Ph.D., TNO Industrial Innovations, Eindhoven, The Netherlands Charles L. H. Hollenkamp, M.D., MBA, Hemologic-Surgical Company, Amersfoort, The Netherlands. Hemologic-Surgical Company contributed to the study by providing medical equipment for data-acquisition on loan.

\section{Compliance with ethical standards}

Conflict of interest This study was funded by the Department of Anesthesiology at the University Medical Center Utrecht. The non- 
profit non-departmental research organization TNO (Netherlands Organization for Applied Scientific Research) provided the prototype of the monitor and has its ownership. For completeness we mention that co-author S. Kossen is an employee at TNO. Hemologic - Surgical Company contributed to the study by providing medical equipment for data-acquisition on loan and had no financial interest with regard to this study.

Open Access This article is distributed under the terms of the Creative Commons Attribution 4.0 International License (http://crea tivecommons.org/licenses/by/4.0/), which permits unrestricted use, distribution, and reproduction in any medium, provided you give appropriate credit to the original author(s) and the source, provide a link to the Creative Commons license, and indicate if changes were made.

\section{References}

1. Hillman K, Bristow P, Chey T, Daffurn K, Jacques T, Norman SL, et al. Antecedents to hospital deaths. Intern Med J. 2001;31:343-8.

2. Franklin C, Mathew J. Developing strategies to prevent inhospital cardiac arrest: analyzing responses of physicians and nurses in the hours before the event. Crit Care Med. 1994;22(2):244-7.

3. Goldhill DR, White SA, Sumner A. Physiological values and procedures in the $24 \mathrm{~h}$ before ICU admission from the ward. Anaesthesia. 1999;54(6):529-34.

4. Buist M, Bernard S, Nguyen TV, Moore G, Anderson J. Association between clinically abnormal observations and subsequent in-hospital mortality: a prospective study. Resuscitation. 2004;62(2):137-41.

5. Fieselmann JF, Hendryx MS, Helms CM, Wakefield DS. Respiratory rate predicts cardiopulmonary arrest for internal medicine inpatients. J Gen Intern Med. 1993;8(7):354-60.

6. Cuthbertson BH, Boroujerdi M, McKie L, Aucott L, Prescott G. Can physiological variables and early warning scoring systems allow early recognition of the deteriorating surgical patient? Crit Care Med. 2007;35(2):402-9.

7. Jonsson T, Jonsdottir H, Möller AD, Baldursdottir L. Nursing documentation prior to emergency admissions to the intensive care unit. Nurs Crit Care. 2011;16(4):164-9.

8. McBride J, Knight D, Piper J, Smith GB. Long-term effect of introducing an early warning score on respiratory rate charting on general wards. Resuscitation. 2005;65(1):41-4.

9. Odell M, Victor C, Oliver D. Nurses' role in detecting deterioration in ward patients: systematic literature review. J Adv Nurs. 2009;65(10):1992-2006.
10. Anitori L, de Jong A, Nennie F (2009) FMCW radar for life-sign detection. In: IEEE Radar Conference Proceedings. p. 1-6. doi:10.1109/RADAR.2009.4976934.

11. World Medical Association. World Medical Association Declaration of Helsinki: Ethical principles for medical research involving human subjects: WMA General Assembly, Seoul 2008 [article online], 2008. http://www.wma.net/en/30publications/ 10policies/b3/index.html.pdf. Accessed 23 Oct 2008.

12. Niinisto S. Council recommendation of 12 July 1999 on the limitation of exposure of the general public to electromagnetic fields $(0 \mathrm{~Hz}$ to $300 \mathrm{GHz})$. Off $\mathbf{J}$ Eur Communities L. 1999;199:59-70.

13. Wolff C (2015) Frequency-modulated continuous-wave radar (FM-CW Radar). http://www.radartutorial.eu. Accessed $11 \mathrm{Feb}$ 2015.

14. Cooley JW, Tukey JW. An algorithm for the machine calculation of complex fourier series. Math Comput. 1965;19(90):297-301.

15. Prytherch DR, Smith GB, Schmidt PE, Featherstone PI. ViEWStowards a national early warning score for detecting adult inpatient deterioration. Resuscitation. 2007;81(8):932-7.

16. Bland JM, Altman DG. Agreement between methods of measurement with multiple observations per individual. J Biopharm Stat. 2007;17(4):571-82.

17. Clarke WL, Cox D, Gonder-Frederick LA, Carter W, Pohl SL. Evaluating clinical accuracy of systems for self-monitoring of blood glucose. Diab Care. 1987;10:622-8.

18. Leino K, Mildh L, Lertola K, Seppälä T, Kirvelä O. Time course of changes in breathing pattern in morphine- and oxycodoneinduced respiratory depression. Anaesthesia. 1999;54(9):835-40.

19. Mitsis GD, Governo RJM, Rogers R, Pattinson KTS. The effect of remifentanil on respiratory variability, evaluated with dynamic modeling. J Appl Physiol. 2009;106(4):1038-49.

20. Shamir L, Delaney JD, Orlov N, Eckley DM, Goldberg IG. Pattern recognition software and techniques for biological image analysis. PLoS Comput Biol. 2010;6(11):e1000974.

21. Aardal O, Hammerstad J. (2010) Medical radar literature overview. http://www.ffi.no/no/Rapporter/10-00958.pdf. Accessed 23 Dec 2014.

22. Vasu V, Heneghan C, Sezer S, Arumugam T (2011) Contact-free estimation of respiration rates during sleep. In: ISSC proceedings. p. 1-6. http://usb.issc.ie/proceedings/programme/.

23. Li C, Lin J, Xiao Y. Robust overnight monitoring of human vital signs by a non-contact respiration and heartbeat detector. IEEE Eng Med Biol Soc. 2006;. doi:10.1109/IEMBS.2006.260148.

24. Zhu K, Wong YS, Hong GS. Wavelet analysis of sensor signals for tool condition monitoring: a review and some new results. Int J Mach Tools Manuf. 2009;49:537-53.

25. Aardal $\varnothing$, Paichard Y, Brovoll S, Berger T. Physical working principles of medical radar. IEEE Trans Biomed Eng. 2013; doi:10.1109/TBME.2012.2228263. 